\title{
Molar tooth sign - looking beyond the obvious
}

\author{
N Mahomed, ${ }^{1}$ MB BCh, FCRad (SA), S P Prabhu, ${ }^{2}$ MB BS, FRCR \\ ${ }^{1}$ Department of Radiology, University of the Witwatersrand, Johannesburg, South Africa \\ ${ }^{2}$ Department of Radiology, Boston Children's Hospital, Harvard Medical School, Boston, MA, USA
}

Corresponding author: N Mahomed (nasreen.mahomed@wits.ac.za)

The molar tooth sign was initially identified in Joubert syndrome, named after Marie Joubert who first described it in 1968 as a rare autosomal recessive disorder characterised by the neuroradiological hallmark of the molar tooth sign caused by cerebellar vermian hypoplasia. Subsequently, it emerged that the molar tooth sign encompasses many syndromes that are now grouped together and termed Joubert syndrome and related disorders (JSRDs). Knowledge of the newer classification system and the subtypes is important and helps to direct and interpret imaging studies based on clinical signs so as to avoid delay in diagnosis of the hepatic, oculorenal and renal subtypes of JSRDs in patients in whom the molar tooth sign is identified on brain MRI.

S Afr J Rad 2013;17(3):102-103. DOI:10.7196/SAJR.899

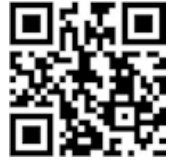

The molar tooth sign refers to the characteristic appearance on axial CT or MRI of enlarged and horizontally directed columnar structures on each side of the midline flanking a deep interpeduncular fossa. ${ }^{[1]}$ This appearance is a result of absence or hypoplasia of the cerebellar vermis, lack of normal dorsal decussation and consequent enlargement of the superior cerebellar peduncles which follow a more horizontal course as they extend perpendicularly to the brainstem between the midbrain and the cerebellum. ${ }^{[1,2]}$ The absence of decussation of the superior cerebellar peduncular fibre tracts causes decreased anteroposterior diameter of the midbrain and a deep interpeduncular fossa. ${ }^{[1]}$ The 'roots' of the molar tooth are represented by thick, perpendicular, non-decussated superior cerebellar peduncles, while the 'body' of the molar tooth is formed by the cerebral peduncles of the brainstem with a deep interpeduncular fossa (Fig. 1a). ${ }^{[3]}$

Diffusion tensor tractography confirms the failure of superior cerebellar peduncle decussation. ${ }^{[2]}$ Colour-coded functional anisotropic maps evaluate for the presence of a focal red dot anterior to the mesencephalon, adjacent to the interpeduncular fossa, which represents the decussation of these fibre tracts (Fig. 1b). Absence of the focal red dot demonstrates failure of decussation of the fibre tracts within the superior cerebellar peduncles and laterally located deep cerebellar nuclei (Fig. 1c). ${ }^{[4]}$

The molar tooth sign was initially identified in Joubert syndrome. Marie Joubert first described this syndrome in 1968 as a rare autosomal recessive disorder characterised by the neuroradiological hallmark of the molar tooth sign caused by cerebellar vermian hypoplasia. ${ }^{[4,5]}$ Subsequently, it has become clear that the molar tooth sign is a radiological hallmark that encompasses many syndromes that are now grouped together and termed Joubert syndrome and related disorders (JSRDs). ${ }^{[2]}$

The genetic basis of JSRDs is complex and heterogenous owing to the resultant mutations of genes encoding ciliary proteins important in brain and ocular development and renal function. ${ }^{[2]}$ According to the most recent classification, JSRDs are classified into 6 phenotypic subgroups: Pure JS (Fig. 1a); JS with ocular defect; JS with oculorenal defects; JS with hepatic defect (Fig. 2); JS with renal defect (Figs 3a and 3b); and JS with orofaciodigital defects. ${ }^{[6]}$ With the identification of various genetic mutations in each of these subtypes, subsequent classifications will need to incorporate this information. ${ }^{[6]}$ It is important for the radiologist to look for associated findings in the central nervous system and other organs, such as the orbits, liver and kidney, associated with JSRDs. ${ }^{[2]}$

Once the molar tooth sign is identified, the brain MRI scan should be reviewed for other associated abnormalities including abnormal configuration and malrotation of the hippocampi, callosal dysgenesis, malformations of cortical development and cephaloceles, which have been described in a sizeable number of patients with JSRDs. ${ }^{[7]}$

Knowledge of the newer classification system and the subtypes is important as it helps to direct and interpret imaging studies based on clinical signs to avoid delay in diagnosis of the hepatic, oculorenal and renal subtypes of JSRDs in patients in whom the molar tooth sign is identified on brain MRI.

1. McGraw P. The molar tooth sign. Radiology 2003;229:671-672. [http://dx.doi.org/10.1148/radiol.2293020764]

2. Barkovich AJ, Raybaud C. Pediatric Neuroimaging, 5th ed. Philadelphia, USA: Lippincott Williams and Wilkins, 2012:481-483.

3. Alorany I, Sabir S, Seidahmed Z, Farooqu H, Salih M. Brainstem and cerebellar findings in Joubert syndrome. J Comput Assist Tomogr 2006;30(1):116-121.

4. Poretti A, Boltshauser E, Loenneker T, et al. Diffusion tensor imaging in Joubert syndrome. Am J Neuroradiol 2007;28(10):1929-1933.

5. Sarikaya B, Akpinar E, Karli-Ogus K, Cil B. Joubert syndrome MRI findings. Neuroanatomy 2004;1:330331.

6. Brancati F, Dallapiccola B, Valente EM. Joubert Syndrome and related disorders. Orphanet Journal of Rare Diseases 2010;5:20. [http://dx.doi.org/10.1186/1750-1172-5-20]

7. Şenocak EU, Oğuz KK, Haliloğlu G, Topçu M, Cila A. Structural abnormalities of the brain other than molar tooth sign in Joubert syndrome-related disorders. Diagn Interv Radiol 2010;16(1):3-6. [http:// dx.doi.org/10.4261/1305-3825.DIR.2673-09.1] 


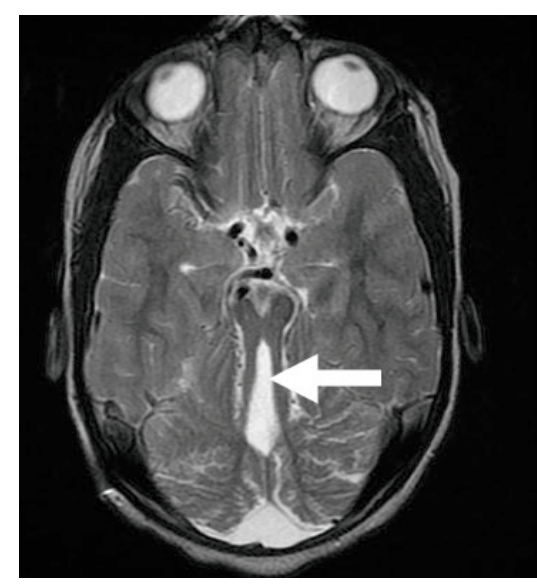

Fig. 1a. Axial T2 MRI at the level of the midbrain demonstrating the molar tooth sign (i.e. thickened superior cerebellar peduncles (arrow indicates left-side peduncle) with a deep interpeduncular fossa) in an 8-year-old patient diagnosed with Joubert syndrome in combination with clinical and imaging findings. This appearance is characteristic of non-decussation of the superior cerebellar peduncles. There is marked vermian hypoplasia.

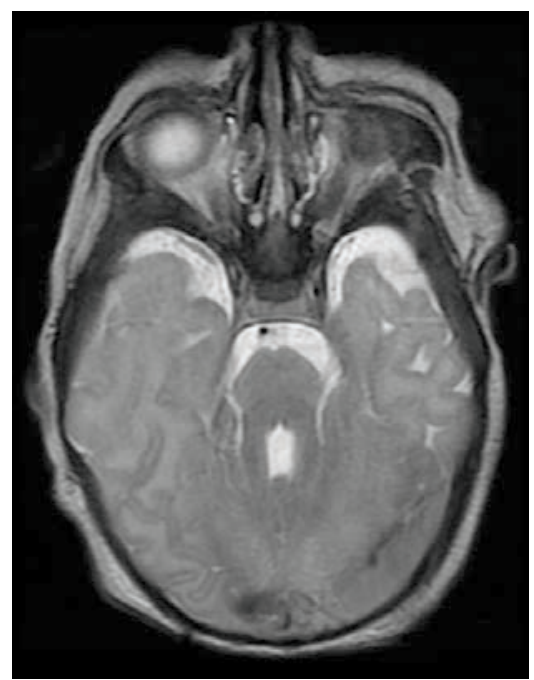

Fig. 2. Joubert syndrome demonstrating the molar tooth sign on axial T2 MRI in a 10-yearold boy with chronic liver disease being treated for Wilson disease with chelation therapy. In view of oculomotor apraxia and presence of the molar tooth sign on his brain MRI, JSRD was diagnosed. Genetic evaluation revealed two mutations for Joubert syndrome and, based on the presence of liver disease, diagnosis of the hepatic variant of JSRD was made.

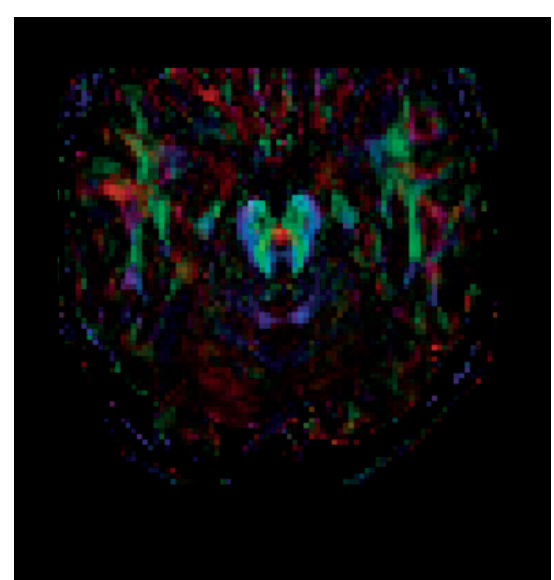

Fig. 1b. MRI 2D tractography in a normal child. Colour-coded functional anisotropic maps demonstrate the presence of a focal red dot anterior to the mesencephalon, adjacent to the interpeduncular fossa which represents the decussation of these fibre tracts.

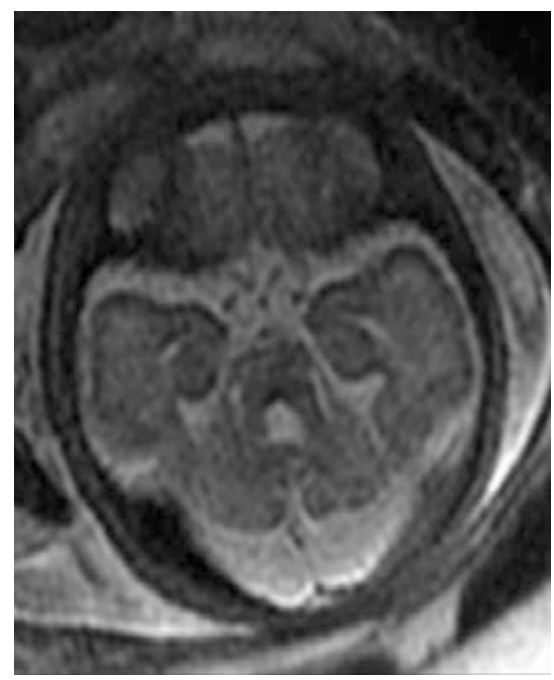

Fig. 3a. Fetal MRI, axial T2, at 29 weeks' gestation demonstrating the molar tooth sign, which was confirmed on postnatal MRI and genetic evaluation.

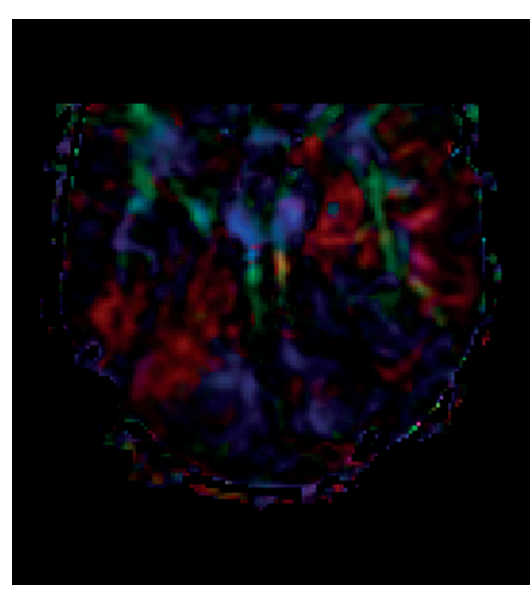

Fig. 1c. MRI 2D tractography in a patient with JSRD demonstrates lack of superior cerebellar peduncle decussation, i.e. the absent focal red dot.

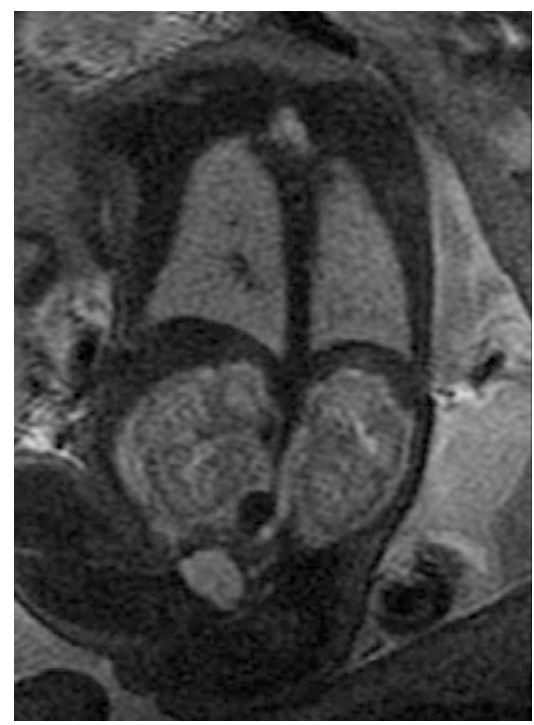

Fig. 3b. Fetal MRI, coronal T2, in the same fetus, demonstrating enlarged echogenic kidneys. This finding in conjunction with the molar tooth sign is consistent with a diagnosis of the JSRD renal subtype. 